\title{
Interview with Gert Storm
}

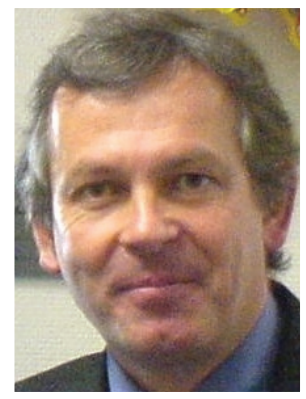

Gert Storm studied biology at the Utrecht University, The Netherlands, and obtained his PhD degree in 1987 at the Department of Pharmaceutics of the same university. He is now Professor of targeted drug delivery at the University of Utrecht, as well as Professor of targeted therapeutics at the MIRA institute of the University of Twente (Enschede, The Netherlands). $\mathrm{He}$ also holds a position at the University Medical Center Utrecht within the Centre for Image-Guided Oncological Interventions. Among various other commitments, Professor Storm is Director of the Drug Delivery program embedded within the new nano initiative, titled NanoNextNL, a member of the Board of Scientific Advisors of the Controlled Release Society, and a Scientific Board member of Enceladus Pharmaceuticals BV, a spin-off company based in Amsterdam (The Netherlands). Gert Storm spoke to Therapeutic Delivery regarding his career to date, his research within the targeted drug-delivery field, and the obstacles that need to be tackled in order to advance this exciting research area.

Interview conducted by Hannah Coaker, Assistant Commissioning Editor.

2 How did you become involved in the field of targeted therapy?

This is very simple; my PhD degree was obtained in Utrecht (The Netherlands) in 1987, based on a thesis regarding doxorubicin-containing liposomes. Soon after that, I did a sabbatical at Liposome Technology Inc. (CA, USA), a company that in those days developed Doxil, the first US FDA-approved liposomal product on the market.

Can you update us on your research group's current work?

At the moment, I am occupying three positions in The Netherlands. For $60 \%$ of my time I am at the Utrecht University (Utrecht, The Netherlands), for 20\% at the University Medical Centre Utrecht hospital (UMCU; Utrecht, The Netherlands), and the other 20\% I am at the University of Twente (Enschede, The Netherlands), in the east of the country. The common denominator for these three research groups is 'targeted nanomedicine', and together they provide an excellent environment for translational drug-targeting research.

All three research teams are well connected, provide an excellent 'from bench-to-clinic' infrastructure, but also have their own identity. Let us start with the Utrecht University (Department of Pharmaceutics), which was for the biggest part of my scientific life and still is my main employer. My main focus has always been on drug targeting, with liposomes designed for this purpose still being popular in my research. However, over the years the targeted drug-delivery system (DDS) arsenal has been broadened considerably, with systems based on biodegradable polymers, peptides and proteins getting much attention, in close collaboration with my colleague Professor Hennink, a synthetic chemist and more of a 'pharmaceutical technologist', whereas I studied biology and developed more as a 'biopharmacist'. For a long time, together with the recently retired Professor Crommelin, the 'pharmacist', we have operated as a fruitful team covering the multidisciplinary aspects of advanced drug-delivery research. A current vital part of our research is the development of triggered release systems. We try to do that by using thermosensitive DDS formulations in combination with ultrasound technology that can heat up tissues in vivo. Triggered release is a very important issue because many targeted nanomedicines often face the problem that if they arrive at the target site the drug is still within the nanocarrier and is not being released efficiently. Triggered release approaches aim to making the drug therapeutically available within the target site, and should lead to better therapeutic efficacy. In close collaboration with the UMCU hospital, a large project is currently in progress studying the clinical application of MRI-guided high-intensity focused ultrasound (MRg-HIFU) in combination with a

\section{Gert Storm}

Utrecht University, Department of Pharmaceutics, Utrecht,

The Netherlands

Tel.: +31 302527288

E-mail: g.storm@uu.nl 


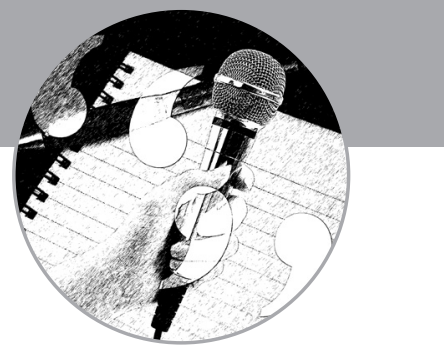

targeted thermosensitive doxorubicin-containing liposome formulation for locoregional cancer treatment. This unique project on demonstrating the functionality of hyperthermia-induced triggered release in the patient is sponsored by the Dutch public-private partnership organization Center for Translational Molecular Medicine (CTMM, Eindhoven, The Netherlands), Philips (Eindhoven, Netherlands) and Celsion (NJ, USA). A second current focus of our research is targeted anti-inflammatory drug formulations. Many of the nanomedicines developed in the past have focused on cancer treatment and anti-inflammatory applications have been somehow a bit neglected. So we work on the design and evaluation of liposomal nanomedicines for the treatment of, for example, rheumatoid arthritis, inflammatory bowel disease, multiple sclerosis, eye inflammation and atherosclerotic plagues. A spin-off company (Enceladus Pharmaceuticals BV, Naarden, The Netherlands) has been created to forward such formulations to the clinic and the market. Translational work is also embedded within national public-private partnerships (e.g., NanoNextNL) and EC-sponsored projects. We recently became involved in two new EC projects on the inhaled delivery of liposomal antibiotics and antimicrobial peptides for targeting serious bacterial lung infections. Also in the context of EC-sponsored research, we contribute to a large nanotoxicology project with the development of safety assays to address potential adverse effects (e.g., complement activation-related pseudoallergy reactions as reported by Professor Szebeni in Budapest) towards intravenously administered particulate nanomedicines in patients. In the wake of liposomes, polymeric micelles for solubilization and targeted delivery of hydrophobic drugs (e.g., paclitaxel) are in focus. Particular attention is also given to delivery systems for nucleic acids exploiting lipid- and polymer-based systems, for example, neovasculature-targeted miRNA/siRNA nanoparticles and decationized polyplexes.

At the UMCU, as just mentioned, I am working in a team that focuses on HIFU applications such as the high temperature ablation of tumor cells, and for mild hypothermia, which entails raising the temperature in tumors by up to approximately $42^{\circ} \mathrm{C}$ and then administering a themosensitive nanomedicine that will leak all the drug in the tumor because of the higher local temperature. Clinical translation of HIFU-mediated ablation is ongoing for patients with breast cancer and with bone metastases in case of prostate cancer. The mild hyperthermia-thermosensitive liposomes approach will be evaluated in patients with breast cancer to obtain proof-of-principle. This approach is MRI-guided, with MRI serving to identify the location of the tumor but also to determine the intratumoral temperature via acquisition of temperature maps. Sonoporation, that is, the combined use of microbubbles and ultrasound to 'permeabilize' endothelial linings is being explored for delivery across the blood-brain barrier. The UMCU team working on all these imaging and imaging-guided drug delivery applications is embedded within the Center for Image Guided Oncological Interventions (CIGOI, also named CBOI in Dutch).

Finally, I am also active in the MIRA Institute for Biomedical Technology and Technical Medicine at the University of Twente. The University of Twente is a relatively young university with proven excellence in nanotechnological science, and aims, with the Targeted Therapeutics group, to strengthen the application of nanotechnology for improving healthcare. A major line of research in this new group is the development of a robust and versatile technology to target tumor stroma. One critical aspect often overlooked in tumor targeting approaches is the complex tumor microenvironment: tumor cells do not act alone but in close interaction with tumor stroma surrounding and nourishing the malignant tumor cells. If we can, for example, target the cancer-associated fibroblasts, the most prominent cell type in tumor stroma, we may be able to hit the tumor in its Achilles' heel.

Q Is much of your time working at the UMCU dedicated towards the clinical translation of the delivery systems that you have developed in the laboratory?

Yes. As said, I am active within the CIGOI center of the UMCU hospital. This center has the central objective to provide real-time image guidance for precise treatment of localized tumors. Within the CIGOI a range of minimally invasive image-guided treatment techniques are being developed such as MRI-controlled HIFU, MRI-driven linear accelerator (first prototype installed at UMCU), MRI-guided robotic brachytherapy, and intra-arterial radio-embolization with holmiumloaded microspheres. The combination of this great imaging infrastructure in this hospital with drug-delivery approaches gives us a unique opportunity to make an impact on cancer therapy, and potentially also other diseases. In addition to the UMCU hospital, translational aspects of liposomal formulations are also addressed in other hospitals (in Amsterdam, Nijmegen, Rotterdam, Leiden, 
Leuven, New York and Singapore) for the treatment of rheumatoid artritis, inflammatory bowel disease, atherosclerosis and ophthalmological complications.

What would you consider your most important achievement to date?

I think the encapsulation of corticosteroids in liposomes to treat rheumatoid artritis has been most rewarding. I was also instrumental in bringing the liposomal corticosteroid platform technology to the stage of industrial development, with my former PhD student on this subject (B Metselaar) now being the CEO of the spin-off company focused on this. As a more general achievement, I think I have been quite successful in connecting academia with the clinic and industry to address the clinical translation of academic findings. I was coordinator of the recently finished Integrated Project (FP6), titled MediTrans, on targeted nanomedicines, with a consortium composed of 30 European partners and funded by the EC and industry. Currently, I am director of the Drug Delivery program embedded within the recently approved New Nano Initiative (NanoNextNL), strongly sponsored by the Dutch government and industry. I am also principal investigator of the HIFU-CHEM project sponsored by the Dutch public-private partnership organization CTMM. In addition to facilitating the creation of spin-off companies, I am also keen to keep close contact with the industry, from small to big pharmaceutical companies. In this context of bringing together academic, clinical and industrial scientists, it is also worth mentioning that I was involved in the foundation and am currently on the board of the European Clinical Nanomedicine Society (CLINAM, Basel, Switzerland).

As a member of the Board of Scientific Advisors of the Controlled Release Society, you highlight the need to create a strong link between the community of pharmaceutical scientists and the community of imaging probes developers to boost the emerging field of imaging-guided drug delivery. Do you think the necessary steps are being made to achieve this goal?

Yes, I think so. In Europe this development was boosted by the EC-sponsored MediTrans project in which imaging and drug delivery really came together. MediTrans was based on a consortium representing a good mix of imaging and pharmaceutical partners. As a sort of follow-up, Professor Aime from Turin (Italy) has applied for additional EC funding (Research Concerted Action of EU), and now this COST program has been approved and running under the title 'Theranostics Imaging and Therapy'. Recently meetings were held in Turin and Athens (Greece). I see the imaging-guided drug delivery field growing further, with separate sessions now at major conferences such as the European Society for Molecular Imaging (Turin) and the World Molecular Imaging Congress (GA, USA) earlier this year. I expect that next year separate sessions will be organized at the Controlled Release Society in Chicago, USA and European Clinical Nanomedicine Society.

Q Do you think advances in targeted delivery are significant in achieving personalized delivery to patients?

Yes, I do. In particular for targeted theranostic modalities, which combine an imaging ('see') and drug ('treat') component. The 'see' part is to monitor the localization of the drug-delivery system, to assess whether the carrier-associated drug is liberated efficiently within the target site, and to get a feeling whether the released drug exerts activity as well. Let me give a relatively simple, straightforward example to illustrate the first aspect of target localization. The liposomal nanomedicine Doxil is a blockbuster and its approval is primarily based on demonstration of reduced side effects ('site-avoidance delivery'), rather than on demonstration of superior efficacy ('site-specific delivery'). There is a serious concern that Doxil is not always accumulating sufficiently in the patient's tumor. Therefore, if there were to be a prescreening imaging step first to 'see' whether the nanomedicine indeed accumulates after intravenous administration, followed by a 'treating' step only in case of positive accumulation data, this would be a valuable personalized delivery approach. In this way you are not burdening many patients with potentially toxic drugs if there is evidence that the tumor is not able to take the drug, and at the same time the chance for achieving antitumor efficacy is substantially increased.

Your most recent work focuses on the application of HIFU in targeted drug delivery. What do you think is the potential of this technique?

This, I think, has great potential for treating certain local pathologies. We are trying to develop oncological interventions for better control of local tumors, such as liver tumors such as hepatocellular 


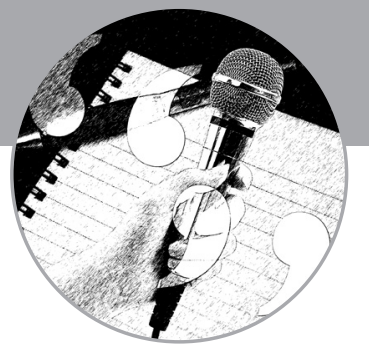

carcinoma or liver metastases originating from colorectal tumors, bone metastases in case of prostate cancer, brain, kidney and breast tumors. The tumors that we look at are often inoperable, or at least complete surgery is not possible. Also tissue transplantation is often not an option because the patient is too weak. As already mentioned earlier, one can aim for ablation (heating tumor tissue up to temperatures above $60^{\circ} \mathrm{C}$ ) and/or for mild hyperthermia (up to $42^{\circ} \mathrm{C}$ ) in combination with targeted thermosensitive nanomedicines. The HIFU technique is not applicable to all sites of malignant growth, due to the inability to reach certain body areas efficiently with highly energetic ultrasound waves without harming healthy tissue. However, the MRI-guided HIFU technology is progressing well in the clinic for thermal ablation of uterine fibroids and some of the tumor types just described. Its more recent application to trigger 'on demand' the release of drug from temperature-sensitive nanomedicines once they have entered the tumor area, is now receiving dedicated attention, and we expect to start a first clinical proof-of-concept study in breast cancer patients in 2014. In addition, its use in combination with microbubbles, which are vesicles filled with gas, to open up anatomical barriers, like the blood-brain barrier, is appealing as well.

Q As Program Director of a new government and industry initiative, known as NanoNextNL, what is your vision in this role?

Simply, to intensify structural collaborations between academia, industry and the clinic, in order to drive the nanomedicine field to yield more pharmaceutical products that are profitable for the society. This simple vision is clearly reflected in the composition of the consortium carrying out the Drug Delivery program within NanoNextNL: 9 hospitals, 16 industries and 5 universities.

Q How do you ensure a healthy collaboration between industry and academia? Do you think that intellectual property-related matters can sometimes hinder this partnership? Nowadays both industry and academia are more eager to collaborate, as they need each other more and more in order to move forward. It goes without saying that one needs to create an atmosphere of mutual trust and understanding, and that confidentiality and intellectual property issues regarding background and foreground intellectual property are satisfactorily arranged to safeguard particularly the industrial interests. But a lot of experience is out there, for example within public-private organizations, to arrange all this properly and efficiently via formal agreements.

Q Do you find that promising research is often not pursued due to a lack of funding from the big pharmaceutical companies?

This is indeed true, it is often a matter of money and what can you do? I am trying to bridge this gap and I think many academics, now and in the past, work in too isolated a manner. Networking with clinicians and industrial scientists gives more opportunities to push promising research further. In my country, public-private partnership organizations (NanoNextNL, CTMM, TI Pharma and BMM) have been and are still very popular. But government budget cuts bring the sustainability of these organizations into great danger. We are presently facing this problem, but I am convinced that after some years new possibilities and ways of funding will be found. For the time being, the increasing interest of pharmaceutical companies to collaborate with academia for medicine development provides already exciting opportunities.

Q How do you anticipate the field of targeted delivery will progress in the next 5-10 years? It is perhaps not nice to say but I expect 'more of the same'. To make real next steps forward, we need a much better understanding of the biological obstacles we are facing and of the ways that we can tackle these barriers. Nevertheless, much of the currently available targeted delivery knowledge is really valuable and not fully exploited yet for further translation towards the clinic. Therefore, I do hope that clinical translation efforts will continue, to compensate for the sad but true message of a recent paper with the title 'Cancer nanomedicines: so many papers and so few drugs'. Pharmaceutical development, GMP and safety issues, by far from trivial issues, will require proper attention for realizing this hope. I expect that the 'targeted delivery community' will address more and more diseases other than cancer, for example chronic inflammation and also 'easy' accessible targets, such as the eye. It should also be realized that most of the particulate nanomedicines that are on the market have been approved on the basis of their favorable safety profile rather than their superior efficacy. So, I think the field will try to improve the efficacy and activity of nanomedicines, 
as exemplified by the HIFU/thermosensitive liposome approach just discussed. Image-guided drug delivery will be increasingly important to monitor the complete targeted drug-delivery process, which is needed in order to be able to optimize the nanomedicine in question. Also combination treatments, for example, more drugs in one nanomedicine or combination with modalities such as radiotherapy and ultrasound, will become more popular. Certainly, we should not forget about the monoclonal antibodies and antibody-drug conjugates: following a big dip, they are now very successful and will continue to catch much attention.

The next 5-10 years will also see continued efforts towards targeted biotherapeutics, such as siRNA, miRNA, pDNA and therapeutic proteins. The development of such targeted formulations is quite complex, and it will likely be needed to obtain better control over the intracellular fate of such systems and in turn acheive improved control over gene and protein expression. In addition to established systems like liposomes and polymeric micelles, novel bio-inspired DDS are emerging, such as the exosomes/microvesicles, endogenous nanocarriers naturally secreted by mammalian cells, which are claimed to possess exciting natural targeting capabilities. In the latter case, clearly more in-depth understanding of their pharmacokinetics, biodistribution and targeting capacity is highly needed in order to be able to realistically assess their potential as drug-delivery vehicles. Nanoformulations targeting cells of the immune system, such as dendritic cells, will also be embraced by the delivery field for vaccination purposes, particularly to fight cancer.

What words of advice would you give to a young scientist envisaging a career in this area? Never give up. Young people ask themselves pragmatic questions, particularly in these more difficult years of funding and jobs: what can I do, shall I become a postdoc? Can I become a Professor? They feel that the possibility to get an academic research career is not guaranteed, and therefore they may give up too soon. I would say: try not to follow the easy path, and face the uncertainties of the future. Your struggles will develop your strengths. Be ambitious, think international, and develop yourself in research groups of high quality (other than the group where you did your PhD). Do not think what science can do for you, but what you can do for science.

\section{Disclaimer}

The opinions expressed in this interview are those of the interviewee and do not necessarily reflect the views of Future Science Ltd.

\section{Financial \& competing interests disclosure}

The interviewee has no relevant affiliations or financial involvement with any organization or entity with a financial interest in or financial conflict with the subject matter or materials discussed in the manuscript. This includes employment, consultancies, honoraria, stock ownership or options, expert testimony, grants or patents received or pending, or royalties. No writing assistance was utilized in the production of this manuscript. 